\title{
Classification of Six-Point Metrics
}

\author{
Bernd Sturmfels and Josephine Yu \\ Department of Mathematics \\ University of California \\ Berkeley, CA 94720 \\ [bernd, jyu] @math . berkeley . edu
}

Submitted: Mar 10, 2004; Accepted: Jun 7, 2004; Published: Jul 1, 2004

MR Subject Classifications: Primary 51K05, 52B45; Secondary 05C12

\begin{abstract}
There are 339 combinatorial types of generic metrics on six points. They correspond to the 339 regular triangulations of the second hypersimplex $\Delta(6,2)$, which also has 14 non-regular triangulations.
\end{abstract}

\section{The Metric Fan}

We consider the cone of all metrics on the finite set $\{1,2, \ldots, n\}$ :

$$
C_{n}=\left\{d \in \mathbb{R}^{\left(\begin{array}{l}
n \\
2
\end{array}\right)}: d_{i j} \geq 0 \text { and } d_{i j}+d_{j k} \geq d_{i k} \text { for all } 1 \leq i, j, k \leq n\right\} .
$$

This is a closed convex pointed polyhedral cone. Its extreme rays have been studied in combinatorial optimization $[4,5]$. Among the extreme rays are the splits. The splits are the metrics $\sum_{i \in A} \sum_{j \notin A} e_{i j} \in \mathbb{R}^{\left(\begin{array}{c}n \\ 2\end{array}\right)}$ as $A$ ranges over nonempty subsets of $\{1,2, \ldots, n\}$. There is an extensive body of knowledge (see [5,9]) also on the facets of the subcone of $C_{n}$ generated by the splits.

Our object of study is a canonical subdivision of the metric cone $C_{n}$. It is called the metric fan and denoted $M F_{n}$. A quick way to define the metric fan $M F_{n}$ is to say that it is the secondary fan of the second hypersimplex

$$
\Delta(n, 2)=\operatorname{conv}\left\{e_{i}+e_{j}: 1 \leq i<j \leq n\right\} \quad \subset \quad \mathbb{R}^{n} .
$$

Every metric $d$ defines a regular polyhedral subdivision $\Delta_{d}$ of $\Delta(n, 2)$ as follows. The vertices of $\Delta(n, 2)$ are identified with the edges of the complete graph $K_{n}$, and subpolytopes of $\Delta(n, 2)$ correspond to arbitrary subgraphs of $K_{n}$. A subgraph $G$ is a cell of $\Delta_{d}$ if there exists an $x \in \mathbb{R}^{n}$ satisfying

$$
x_{i}+x_{j}=d_{i j} \text { if }\{i, j\} \in G \quad \text { and } \quad x_{i}+x_{j}>d_{i j} \text { if }\{i, j\} \notin G .
$$


Two metrics $d$ and $d^{\prime}$ lie in the same cone of the metric fan $M F_{n}$ if they induce the same subdivision $\Delta_{d}=\Delta_{d^{\prime}}$ of the second hypersimplex $\Delta(n, 2)$. We say that the metric $d$ is generic if $d$ lies in an open cone of $M F_{n}$. This is equivalent to saying that $\Delta_{d}$ is a regular triangulation of $\Delta(n, 2)$.

These triangulation of $\Delta(n, 2)$ and the resulting metric fan $M F_{n}$ were studied by De Loera, Sturmfels and Thomas [3], who had been unaware of an earlier appearance of the same objects in phylogenetic combinatorics $[1,6]$. In [6], Dress considered the polyhedron dual to the triangulation $\Delta_{d}$,

$$
P_{d}=\left\{x \in \mathbb{R}_{\geq 0}^{n}: x_{i}+x_{j} \geq d_{i j} \text { for } 1 \leq i<j \leq n\right\},
$$

and he showed that its complex of bounded faces, denoted $T_{d}$, is a natural object which generalizes the phylogenetic trees derived from the metric $d$. Both [3] and [6] contain the description of the metric fans $M F_{n}$ for $n \leq 5$ :

- The octahedron $\Delta(4,2)$ has three regular triangulations $\Delta_{d}$. They are equivalent up to symmetry. The corresponding tight span $T_{d}$ is a quadrangle with an edge attached to each of its four vertices. The three walls of the fan $M F_{4}$ correspond to the trees on $\{1,2,3,4\}$.

- The fan $M F_{5}$ has 102 maximal cones which come in three symmetry classes. The tight spans $T_{d}$ of these three metrics are depicted in [6, Figure A3], and the corresponding triangulations $\Delta_{d}$ appear (in reverse order) in [3, page 414]. For instance, the thrackle triangulation of $[3, \S 2]$ corresponds to the planar diagram in [6]. All three tight spans $T_{d}$ have five two-cells. (The type $T_{X, D_{3}}$ is slightly misdrawn in [6]: the two lower left quadrangles should form a flat pentagon).

The aim of this article is to present the analogous classification for $n=6$. The following result was obtained with the help of Rambau's software TOPCOM [13] for enumerating triangulations of arbitrary convex polytopes.

Theorem 1 There are 194, 160 generic metrics on six points. These correspond to the maximal cones in $M F_{6}$ and to the regular triangulations of $\Delta(6,2)$. They come in 339 symmetry classes. The hypersimplex $\Delta(6,2)$ has also 3,840 non-regular triangulations which come in 14 symmetry classes.

This paper is organized as follows. In Section 2 we describe all 12 generic metrics whose tight span $T_{d}$ is two-dimensional, and in Section 3 we describe all 327 generic metrics whose tight span has a three-dimensional cell. Similarly, in Section 4, we describe the 14 non-regular triangulations of $\Delta(6,2)$. In each case a suitable system of combinatorial invariants will be introduced. In Section 5 we study the geometry of the metric fan $M F_{6}$. The rays of $M F_{6}$ are precisely the prime metrics in [12]. We determine the maximal cones incident to each prime metric, and we discuss the corresponding minimal subdivisions of $\Delta(6,2)$. In Section 6 we present a software tool for visualizing the tight span $T_{d}$ of any finite metric $d$. This tool was written written in POLYMAKE [10] with the help of 
Michael Joswig and Julian Pfeifle. We also explain how its output differs from the output of SPLITSTREE [8].

A complete list of all six-point metrics has been made available at

$$
\text { bio.math. berkeley.edu/SixPointMetrics }
$$

For each of the $339+14$ types in Theorem 1, the regular triangulation, Stanley-Reisner ideal, and numerical invariants are listed. The notation is consistent with that used in the paper. In addition, the webpage contains interactive pictures in JAVAVIEW [11] of the tight span of each metric.

\section{The 12 Two-Dimensional Generic Metrics}

We identify each generic metric $d$ with its tight span $T_{d}$, where the exterior segments have been contracted $^{1}$ so that every maximal cell has dimension $\geq 2$. With this convention, generic four-point metrics are quadrangles and five-point metrics are glued from five polygons (cf. [6, Figure A3]). The generic six-point metrics, on the other hand, fall naturally into two groups.

Lemma 2 Each generic metric on six points is either a three-dimensional cell complex with 26 vertices, 42 edges, 18 polygons and one 3-cell, or it is a two-dimensional cell complex with 25 vertices, 39 edges and 15 polygons. There are 327 three-dimensional metrics and 12 two-dimensional metrics.

We first list the twelve types of two-dimensional metrics. In each case the tight span consists of 15 polygons which are either triangles, quadrangles or pentagons. Our first invariant is the vector $B=\left(b_{3}, b_{4}, b_{5}\right)$ where $b_{i}$ is the number of polygons with $i$ sides. The next two invariants are the order of the symmetry group and the number of cubic generators in the Stanley-Reisner ideal of the triangulation $\Delta_{d}$. The last item is a representative metric $d=\left(d_{12}, d_{13}, d_{14}, d_{15}, d_{16}, d_{23}, d_{24}, d_{25}, d_{26}, d_{34}, d_{35}, d_{36}, d_{45}, d_{46}, d_{56}\right)$ :

Type 1: $(1,10,4), 1,2,(9,9,10,13,18,18,17,6,11,17,14,9,11,8,17)$

Type 2: $(1,10,4), 1,3,(8,8,8,14,15,16,14,6,9,12,12,7,8,7,13)$

Type 3: $(1,10,4), 1,5,(5,6,7,8,12,11,10,5,7,11,6,6,7,5,10)$

Type 4: $(1,10,4), 2,3,(7,5,7,12,12,12,12,5,7,10,9,7,7,5,10)$

Type 5: $(1,10,4), 2,4,(6,7,8,10,14,13,12,6,8,13,9,7,6,6,10)$

Type 6: $(1,10,4), 2,5,(7,7,7,11,14,12,12,6,7,14,10,7,6,7,11)$

Type $7:(1,10,4), 8,6,(5,5,5,8,10,10,8,5,5,8,5,5,5,5,8)$

Type 8: $(2,8,5), 1,3,(5,5,7,10,11,10,10,5,8,10,7,6,5,4,7)$

Type 9: $(2,8,5), 2,4,(7,7,8,10,14,14,13,5,9,13,9,7,10,6,14)$

Type 10: $(2,8,5), 2,4,(5,4,5,8,9,7,8,3,6,9,6,5,5,4,7)$

\footnotetext{
${ }^{1}$ Note that the exterior segments do appear in Figures $1-5$ of this paper and in the diagrams on our webpage. They are drawn in green for extra clarity.
} 
Type 11: $(2,8,5), 2,4,(4,5,5,8,9,9,7,4,7,8,5,4,5,4,7)$

Type 12: $(3,6,6), 12,3,(3,3,5,6,6,6,6,3,5,6,5,3,3,3,6)$

The three metrics of types 9,10 and 11 cannot be distinguished by the given invariants. In Section 5 we explain how to distinguish these three types.

The metric with the largest symmetry group is Type 12. Its symmetry group has order 12. This combinatorial type of this metric is given by the Stanley-Reisner ideal of the corresponding regular triangulation of $\Delta(6,2)$ :

$$
\begin{gathered}
\left\langle x_{36} x_{14}, x_{25} x_{34}, x_{35} x_{46}, x_{16} x_{45}, x_{35} x_{12}, x_{26} x_{35}, x_{36} x_{45}, x_{15} x_{36}, x_{26} x_{45}, x_{12} x_{46},\right. \\
x_{12} x_{56}, x_{25} x_{36}, x_{45} x_{23}, x_{24} x_{13}, x_{45} x_{12}, x_{34} x_{12}, x_{25} x_{46}, x_{23} x_{46}, x_{16} x_{25}, x_{13} x_{46}, \\
x_{24} x_{36}, x_{35} x_{14}, x_{13} x_{56}, x_{26} x_{14}, x_{26} x_{13}, x_{15} x_{46}, x_{36} x_{12}, x_{45} x_{13}, x_{25} x_{14}, x_{25} x_{13}, \\
\left.x_{15} x_{26} x_{34}, x_{23} x_{56} x_{14}, x_{16} x_{24} x_{35}\right\rangle .
\end{gathered}
$$

The number of quadratic generators is 30 , and this number is independent of the choice of generic metric. The number of cubic generators of this particular ideal is three (the last three generators), which is the third invariant listed under "Type 12". These cubic generators correspond to "empty triangles" in the triangulation $\Delta_{d}$. For instance, the cubic $x_{15} x_{26} x_{34}$ means that $\operatorname{conv}\left\{e_{1}+e_{5}, e_{2}+e_{6}, e_{3}+e_{4}\right\}$ is not a triangle in $\Delta_{d}$ but each of its three edges is an edge in $\Delta_{d}$. In the tight span $T_{d}$ this can be seen as follows:

\section{\{geodesics between 1 and 5$\} \cap$ \{geodesics between 2 and 6 \} \\ $\cap\{$ geodesics between 3 and 4$\}=\emptyset$,}

but any two of these sets of geodesics have a common intersection. This can be seen in the picture of the tight span of the type 12 metric in Figure 1.

The twelve generic metrics listed above demonstrate the subtle nature of the notion of combinatorial dimension introduced in [6]. Namely, the combinatorial dimension of a generic metric $d$ can be less than that of a generic split-decomposable metric [1]. This implies that the space of all $n$-point metrics of combinatorial dimension $\leq 2$ is a polyhedral fan whose dimension exceeds the expected number $4 n-10$ (cf. [7, Theorem 1.1 (d)]).

For six-point metrics, this discrepancy can be understood by looking at the centroid $\left(\frac{1}{3}, \frac{1}{3}, \frac{1}{3}, \frac{1}{3}, \frac{1}{3}, \frac{1}{3}\right)$ of the hypersimplex $\Delta(6,2)$. There are 25 simplices in $\Delta(6,2)$ which contain the centroid: the 15 triangles given by the perfect matchings of the graph $K_{6}$ and the 10 five-dimensional simplices corresponding to two disjoint triangles in $K_{6}$. In any given triangulation $\Delta_{d}$, the centroid can lie in either one or the other. In the former case, the tight span $T_{d}$ has a 3-dimensional cell dual to the perfect matching triangle in $\Delta_{d}$. The combinatorial possibilities of these 3-cells will be explored in Section 3. In the latter case, the tight span $T_{d}$ has a distinguished vertex dual to the two-disjoint-triangles simplex in $\Delta_{d}$. This vertex lies in nine polygons of $T_{d}$ which form a link of type $K_{3,3}$. But there is no 3 -cell in $T_{d}$. The distinguished vertex is the one in the center in Figure 1. 


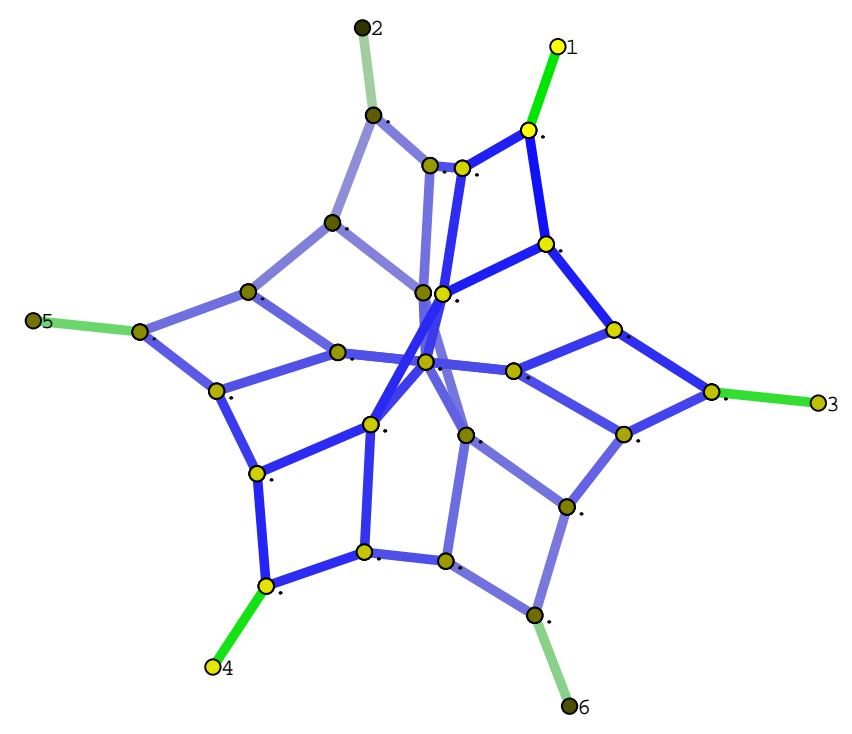

Figure 1: The tight span of the metric \# 12

\section{The 327 Three-Dimensional Generic Metrics}

We next classify the 327 three-dimensional metrics. It turns out that in each case, the unique 3-dimensional cell is a simple polytope, so its numbers $v$ of vertices and $e$ of edges are determined by its number $f$ of faces:

$$
v=2 f-4 \quad \text { and } \quad e=v+f-2 .
$$

We consider the two vectors $R=\left(r_{3}, r_{4}, r_{5}, r_{6}\right)$ and $B=\left(b_{3}, b_{4}, b_{5}, b_{6}\right)$ where $r_{i}$ is the number of polygons with $i$ edges on the 3-cell and $b_{i}$ is the number of polygons with $i$ edges not on the 3-cell. It turns out that no type has a polygon with 7 or more sides. Hence the number of facets of the 3 -cell is $f=r_{3}+r_{4}+r_{5}+r_{6}$. Our third invariant is the pair $S=\left(s_{2}, s_{3}\right)$ where $s_{2}$ (resp. $\left.s_{3}\right)$ is the number of $2 / 4$-splits (resp. 3/3-splits) lying on the cone of the metric fan containing $d$. The fourth invariant is the pair $C=\left(c_{5}, c_{6}\right)$ where $c_{i}$ is the number of cubic generators of the Stanley-Reisner ideal which involve $i$ of the points. And finally we list $(g, t)$ where $g$ is the order of the symmetry group and $t$ is the number of types which share these invariants. These invariants divides the 327 threedimensional metrics into 251 equivalence classes. We order the classes lexicographically according to the vector $(f, R, B, S, C,(g, t))$. The 251 invariants are given in the following long list of strings $R B S C g t$ :

$\begin{array}{llll}40000 a 22600241 & 40000941501111 & 40000941502121 & 40001751401011 \\ 40001751402011 & 40001832501111 & 40001832502111 & 40002642402011 \\ 40002642402021 & 40002642403021 & 40002642404021 & 40002723502121 \\ 40003533404011 & 40004424406081 & 23000 a 21512121 & 23000850401011 \\ 23000850502111 & 23000931402011 & 23000931501111 & \underline{23000940510122}\end{array}$


23000940511113 23001660404021 23001750511113 23002551404012 23003442406021 $06000 a 20620041$ 06000840411011 06000921410021 06000930511011 06001650412012 06001740412011 06001830422011 06002460424041 06003270406041 22200930511113 22201650404021 22202541406021 $05200 a 10520011$ 05200920310011 $\underline{05200920420014}$ 05201640412012 05201730412011 05201820422012 05202540413011 13300830412011 13300920511011 13301730411111 13301820522011 13302540413011 30311640404011 04400910320012 04401720312014 04401720422011 04401810422011 04402530323012 06020820420041 13310820411011 13311720422012 13312530314011 21410820412021 22222440414021 03601710311011 03602700332011 04411710311011 04412520312011 04412610323011
23000940512122 23001741402012 23001750512112 23002560512122 $06000 a 11411011$ $\underline{06000 c 006300 c 1}$ 06000840412021 06000930410011 06001650403021 06001650422011 06001740511011 06001830522011 06002541414021 22200840402011 22200930512113 22201731404011 22203270406041 $05200 b 00530021$ 05200920410011 05200920510021 05201640422011 05201730413011 05201820522011 05202621314021 13300920410111 13301640404011 13301730412011 13302450406021 13302630424021 30312450406021 04400910410011 04401720321014 04401810321014 04401900431011 04402620323012 06021720422011 13310910411011 13311810422011 13312620424021 21412620424041 40042440406081 03601710322011 03602700332021 04411710321011 04412520323011 04412700332021
23000940610121 23001741403011 23001822404021 23002632404011 $06000 a 20420022$ 06000840410011 06000840420022 06000930411011 06001650404021 06001731412011 06001821411011 06002460404011 06002631413011 22200840502121 22201650403021 22201740512113 $05200 a 01310021$ 05200830410011 05200920410021 05200920520021 05201730312011 05201730422012 05202450414011 05202630424021 13300920411012 13301640412013 13301730413012 13302450414011 22210920512122 $04400 a 00420021$ 04400910420011 04401720322011 04401810421012 04402530312012 $06020 a 00420021$ 06022440424041 13311720312012 13312440414011 14120910411111 22221720413011 03600900320021 03602610222013 03603420224023 04411800321012 04412610222011 04413420224021
23001660402011 23001741502111 23001831512111 23002641512121 $06000 a 20520012$ 06000840410021 06000840510021 06000930510012 06001650411011 06001740411011 06001821413011 06002460412021 06002640424041 22200921402021 22201650404011 22202460404011 $05200 a 10420012$ 05200830411011 05200920411011 05201640411011 05201730411011 05201811312011 05202450424021 13300830411011 13300920411111 13301640422011 13301820422011 13302450424021 23020920512121 $04400 a 00430021$ 04401720311012 04401720411012 04401810421022 04402530314011 $06020 a 00430041$ 06022620424041 13311720413011 13312440424021 20602440424041 22222440406041 03600900330061 03602610322013 04410900310021 04411800331011 04412610322011 05221710321011 


$\begin{array}{llll}05221800321012 & 05221800331011 & 05222520312021 & 05222520323011 \\ 05222610323011 & 06030900310161 & 12511710312011 & 12512520323011 \\ 13321710312011 & 13321710322011 & 13322520314011 & 13322520323011 \\ 13322610323012 & 23042520314021 & 02802600222011 & 02802600232021 \\ 02803410223021 & 02803410224021 & 03612600222011 & 03612600232011 \\ 03613410223011 & 03613410224022 & 03613500233011 & 04422600222013 \\ 04422600222022 & 04422600232022 & 04423410223011 & 04423410223023 \\ 04423500233013 & 05232600222021 & 05232600232021 & 05233410224021 \\ 06042600222041 & 12523410224022 & 13333410224021 & 14143410224021 \\ 02814300134012 & 02814300134021 & 03624300134011 & 03624300134021 \\ 04434300134013 & 04434300134021 & 05244300134021 & 02826000046041 \\ 03636000046021 & 03636000046061 & 04446000046083 & \end{array}$

The letters $a, b$ and $c$ appearing in this list represent the integers 10,11 and 12 . We label the 327 types of three-dimensional metrics as Type 13, Type 14, .., Type 339, in the order in which they appear in this list. Whenever the string does not end in a 1 then that string refers to more than one type.

For instance, the first underlined string refers to Type 32 and Type 33. For both of these types, the three-dimensional cell in $T_{d}$ is a triangular prism, hence $R=(2,3,0,0)$. The next invariant $B=(0,9,4,0)$ says that the two-dimensional part of $T_{d}$ consists of nine quadrangles and four pentagons. Types 34 through 38 share these characteristics. What distinguishes Types 32-33 from Types 34-38 is the number of cubic generators in the Stanley-Reisner ideal. The relevant vectors $C=\left(c_{5}, c_{6}\right)$ in the table entries are 01, 11 and 21. The tight spans of Type 32 and 33 are depicted in Figure 2. The location of the four pentagons relative to the six exterior segments of the figure shows that these two types are non-isomorphic.
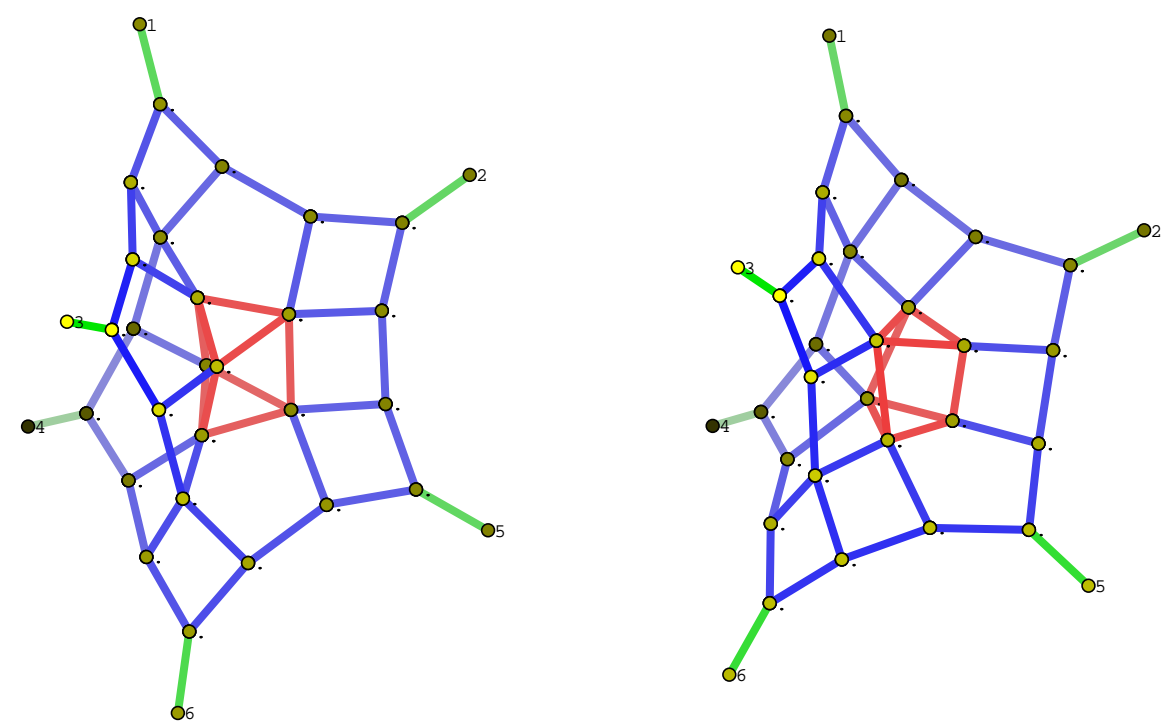

Figure 2: The tight spans of the metrics \# 32 and \# 33

The second underlined string in the long list is Type 66. Its 3-cell is a cube (hence 
$R=0600$ ), and its 2-dimensional part consists of twelve quadrangles (hence $B=0 c 00$ ). This is the unique generic metric which is split-decomposable (hence $S=63$ ) in the sense of [1]. It corresponds to the quadratic Gröbner bases (hence $C=00$ ) and the thrackle triangulation described in $[3, \S 2]$. It has the symmetry group of a regular hexagon (hence $g=12$ ) and it is uniquely characterized by $R$ and $B$ (hence $t=1$ ). Its tight span is the logo for the conference on Phylogenetic Combinatorics which was held in Uppsala, Sweden, in July 2004, http://www.lcb.uu.se/pca04/.

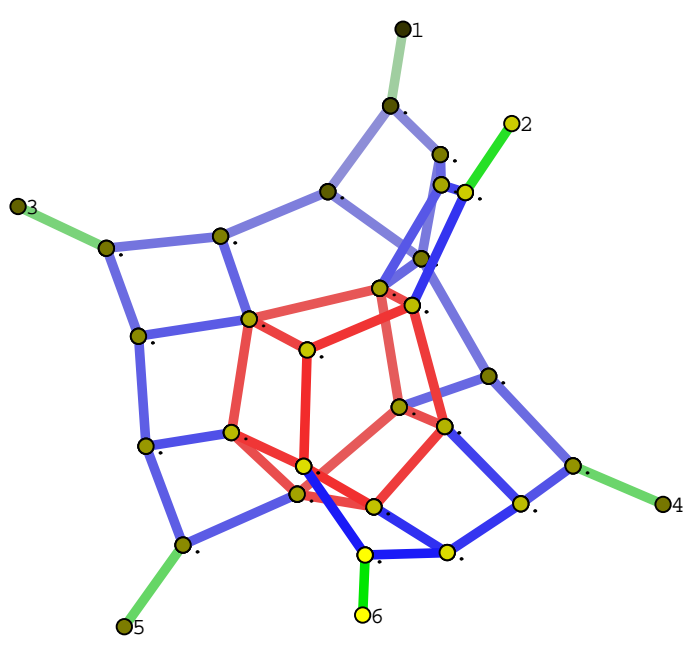

Figure 3: The tight span of the metric \# 131

The third underlined string represents a class of four types, namely, Types 131, 132, 133 and 134. In each of these four cases, the 3-dimensional cell is a pentagonal prism (hence $R=0520$ ), the two-dimensional part consists of nine quadrangles and two pentagons (hence $B=0920$ ), and the Gröbner basis is quadratic (hence $C=00$ ). Figure 3 shows one of these tight spans.

\section{The 14 Non-regular Triangulations}

Theorem 4.2 in [3] states that the hypersimplex $\Delta(n, 2)$ has non-regular triangulations for $n \geq 9$. In this section we strengthen this result as follows.

Theorem 3 The second hypersimplex $\Delta(n, 2)$ admits non-regular triangulations if and only if $n \geq 6$. There are precisely 14 symmetry classes of non-regular triangulations of $\Delta(6,2)$.

It can be shown by explicit computations that all triangulations of $\Delta(4,2)$ and $\Delta(5,2)$ are regular. In what follows we list the 14 non-regular triangulations of $\Delta(6,2)$. Each of them can be lifted to a non-regular triangulation of $\Delta(n, 2)$ for $n \geq 7$ using the technique described at the end of $[3, \S 4]$. 
Each non-regular triangulation $\Delta$ has a dual polyhedral cell complex $T$. This complex $T$ shares all the combinatorial properties of a tight span $T_{d}$, but it cannot be realized as the complex of bounded faces of a polyhedron $P_{d}$. We call $T$ the abstract tight span dual to $\Delta$.

We use the labels Type 340, Type 341, ..., Type 353 to denote the 14 non-regular triangulations $\Delta$ of $\Delta(6,2)$. In each case we describe the abstract tight span $T$. The first four of the 14 abstract tight spans are two-dimensional. They can be characterized by means of the invariants in Section 2:

Type 340: $(0,12,3), 1,1$

Type 341: $(0,12,3), 1,2$

Type 342: $(0,12,3), 6,0$

Type 343: $(1,10,4), 4,1$

The remaining ten abstract tight spans have a unique three-dimensional cell. We characterize them using the invariants $(R, B, S, C, g)$ of Section 3 :

Type 344: $(4,0,0,0),(0,8,6,0),(4,0),(0,0), 4$

Type 345: $(0,4,4,0),(0,8,2,0),(4,0),(0,0), 4$

Type 346: $(0,4,4,0),(2,4,4,0),(4,0),(4,0), 2$

Type 347: $(0,4,4,0),(2,4,4,0),(4,0),(2,0), 4$

Type 348: $(0,4,4,0),(2,4,4,0),(4,0),(2,0), 4$

Type 349: $(0,4,4,0),(2,4,4,0),(4,0),(6,0), 8$

Type 350: $(0,3,6,0),(2,5,2,0),(3,0),(2,0), 2$

Type 351: $(0,3,6,0),(2,5,2,0),(3,0),(4,0), 2$

Type 352: $(0,2,8,0),(2,6,0,0),(2,2),(2,0), 4$

Type 353: $(0,0,12,0),(6,0,0,0),(0,4),(6,0), 24$.

Type \# 353 is the most symmetric one among triangulations $\Delta$ of $\Delta(6,2)$. The corresponding abstract tight span $T$ is a beautiful object, namely, it is a dodecahedron with six triangles and six edges attached, as shown in Figure 4. The Stanley-Reisner ideal corresponding to the dodecahedral type \# 353 is generated by 30 quadrics and 6 cubics. The quadrics in this ideal are

$$
\begin{gathered}
x_{12} x_{35}, x_{12} x_{36}, x_{12} x_{45}, x_{12} x_{46}, x_{12} x_{56}, x_{13} x_{24}, x_{13} x_{26}, x_{13} x_{45}, \\
x_{13} x_{46}, x_{13} x_{56}, x_{14} x_{23}, x_{14} x_{26}, x_{14} x_{35}, x_{14} x_{36}, x_{14} x_{56}, x_{15} x_{23}, \\
x_{15} x_{24}, x_{15} x_{26}, x_{15} x_{36}, x_{15} x_{46}, x_{23} x_{45}, x_{23} x_{46}, x_{23} x_{56}, x_{24} x_{35}, \\
x_{24} x_{36}, x_{24} x_{56}, x_{26} x_{35}, x_{26} x_{45}, x_{35} x_{46}, x_{36} x_{45} .
\end{gathered}
$$

The twelve variables $x_{i j}$ appearing in this list can be identified with the edges of an octahedron. The 30 quadrics are precisely the pairs of disjoint edges of the octahedron. We note that these quadratic generators (and hence the global structure of the tight span) are also shared by the last six types $(334,335,336,337,338,339)$ in the table of Section 3. The simplicial complex represented by these 30 quadrics is (essentially) the boundary of the truncated octahedron (with the six square faces regarded as tetrahedra).

Now, each of the Types 334, 335, 336, 337, 338, 339 and 353 has six cubic generators in its ideal. The choice of these cubic generators amounts to subdividing each of the six 
square faces of the truncated octahedron with one of its two diagonals. Type 353 arises from the most symmetric choice of these diagonals. The six cubics in the ideal for Type 353 are

$$
\underline{x_{34}} x_{12} x_{26}, \underline{x_{34}} x_{15} x_{56}, \underline{x_{16}} x_{23} x_{35}, \underline{x_{16}} x_{24} x_{45}, \underline{x_{25}} x_{13} x_{14}, \underline{x_{25}} x_{36} x_{46} .
$$

The underlined variables are the non-edges of the octahedron.

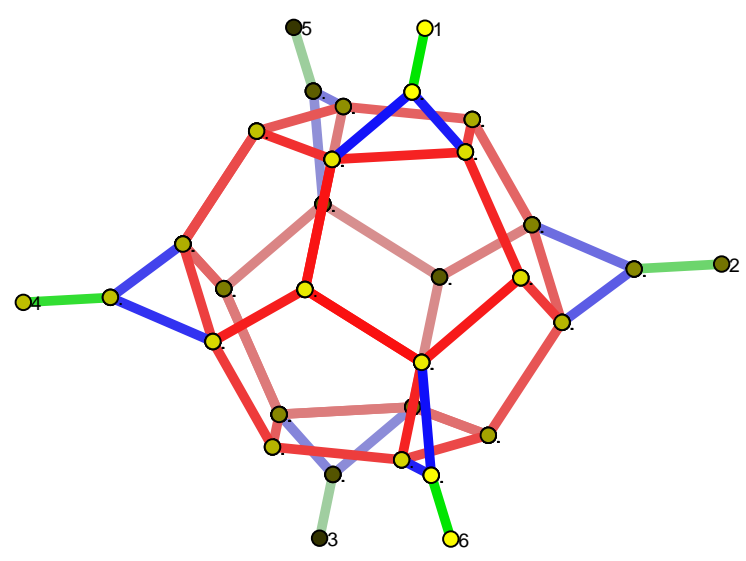

Figure 4: The dodecahedral (abstract) tight span \# 353

The abstract tight span $T$ has the following geometric description. The polytope dual to the truncated octahedron is gotten from the 3-cube by subdividing each of the six facets with a new vertex (thus creating $6 \times 4=24$ edges) and then erasing the 12 edges of the cube. Consider the six 4-valent vertices we just introduced. Each of them can be replaced by two trivalent vertices with a new edge in-between. If this replacement is done in the most symmetric manner then the result is a dodecahedron. Finally, we glue a triangle and an edge on each of the six new edges. The result is Figure 4.

\section{$5 \quad$ Prime Metrics and Minimal Subdivisions}

Koolen, Moulton and Tönges [12] classified all the prime metrics for $n=6$. These are the rays in the metric fan $M F_{6}$. There are 14 symmetry classes:

$$
\begin{aligned}
& \text { 1/5 Split: } d=(0,0,0,0,1,0,0,0,1,0,0,1,0,1,1) \\
& \text { 2/4 Split: } d=(1,1,0,1,1,0,1,0,0,1,0,0,1,1,0) \\
& \text { 3/3 Split: } d=(0,0,1,1,1,0,1,1,1,1,1,1,0,0,0) \\
& \text { Prime } P_{1}: d=(1,1,1,1,2,2,2,2,1,2,2,1,2,1,1) \\
& \text { Prime } P_{2}: d=(1,1,1,2,2,2,2,1,1,2,1,1,1,1,2) \\
& \text { Prime } P_{3}: d=(1,1,1,2,2,2,2,1,1,2,1,1,3,1,2) \\
& \text { Prime } P_{4}: d=(1,1,1,1,2,1,1,1,1,1,1,1,2,1,1) \\
& \text { Prime } P_{5}: d=(1,2,2,2,4,3,3,3,3,2,2,2,4,2,2) \\
& \text { Prime } P_{6}: d=(1,1,2,3,3,2,3,2,2,3,2,2,1,1,2)
\end{aligned}
$$


Prime $P_{7}: d=(1,1,1,2,2,2,2,1,1,2,1,1,2,1,2)$

Prime $P_{8}: d=(1,2,2,4,4,3,3,3,3,4,2,2,2,2,4)$

Prime $P_{9}: d=(1,1,1,2,3,2,2,1,2,2,1,2,3,2,1)$

Prime $P_{10}: d=(0,1,1,1,2,1,1,1,2,2,2,1,2,1,1)$

Prime $P_{11}: d=(0,1,1,2,2,1,1,2,2,2,1,1,1,1,2)$

Our computations provide an independent verification of the correctness and completeness of the results in [12]. Namely, we computed the cone in the metric fan corresponding to each of the 339 metrics. For each cone we computed (using POLYMAKE [10]) the facets and the extreme rays of the cone. And it turned out that the extreme rays we found are precisely the 14 types listed above. All the facets and extreme rays of the 339 types of maximal cones in the metric fan $M F_{6}$ are posted at

\section{bio.math. berkeley.edu/SixPointMetrics}

Our computations lead to the following result.

Proposition 4 If $f_{i}$ is the number of types of maximal cones in the metric fan $M F_{6}$ with $i$ facets and $e_{j}$ is the number with $j$ extreme rays then

$$
\begin{aligned}
\left(f_{15}, f_{16}, \ldots, f_{21}\right) & =(197,42,63,18,8,10,1) \\
\left(e_{15}, e_{16}, \ldots, e_{24}\right) & =(197,60,28,19,20,2,5,2,1,5) .
\end{aligned}
$$

In particular, the metric fan $M F_{6}$ has 197 types of simplicial cones.

Proposition 4 shows that the largest number of facets of any cone is 21, attained by only one type, and the largest number of extreme rays is 24 , attained by five types. The next two examples concern the extremal cases.

Example 5 Type 12 is the last metric listed in the table of Section 2. Its cone in the metric fan is described by the following 21 linear inequalities:

$$
\begin{gathered}
d_{12}+d_{25} \geq d_{15}, \quad d_{13}+d_{36} \geq d_{16}, \quad d_{45}+d_{46} \geq d_{56} \\
d_{25}+d_{45} \geq d_{24}, \quad d_{36}+d_{46} \geq d_{34}, \quad d_{12}+d_{13} \geq d_{23} \\
d_{26}+d_{34} \geq d_{24}+d_{36}, \quad d_{15}+d_{26} \geq d_{16}+d_{25}, \quad d_{14}+d_{23} \geq d_{13}+d_{24} \\
d_{14}+d_{56} \geq d_{16}+d_{45}, \quad d_{16}+d_{35} \geq d_{15}+d_{36}, \quad d_{24}+d_{35} \geq d_{25}+d_{34} \\
d_{14}+d_{23} \geq d_{12}+d_{34}, \quad d_{14}+d_{56} \geq d_{15}+d_{46}, \quad d_{26}+d_{34} \geq d_{23}+d_{46} \\
d_{24}+d_{35} \geq d_{23}+d_{45}, \quad d_{15}+d_{26} \geq d_{12}+d_{56}, \quad d_{16}+d_{35} \geq d_{13}+d_{56} \\
d_{15}+d_{23}+d_{34}+d_{56} \geq d_{16}+d_{24}+2 d_{35} \\
d_{16}+d_{23}+d_{24}+d_{56} \geq d_{15}+2 d_{26}+d_{34} \\
d_{15}+d_{16}+d_{24}+d_{34} \geq 2 d_{14}+d_{23}+d_{56}
\end{gathered}
$$

None of these 21 inequalities is redundant. This cone has 19 extreme rays: the six 1/5 splits, six $2 / 4$ splits, three rays of type $P_{6}$, three rays of type $P_{7}$, and a unique ray of type $P_{2}$, namely, $(1,1,1,2,2,2,2,1,1,2,1,1,1,1,2)$. 
The six 1/6-splits are among the extreme rays of every cone in the metric fan $M F_{6}$. In the next example, we only list the other extreme rays.

Example 6 The five types of cones in $M F_{6}$ with 24 extreme rays are \# 7, \# 26, \# 337, \# 338, and \# 339. Only \# 7 corresponds to a two-dimensional tight span. The following 18 vectors are extreme rays of this cone:

$$
\begin{array}{cc}
2 / 4 \text { split }:(101111000111000) & 2 / 4 \text { split }:(100011110001011) \\
2 / 4 \text { split }:(011111111000000) & 2 / 4 \text { split }:(010011001110011) \\
P_{2}:(111222211211112) & P_{6}:(121333222311222) \\
P_{6}:(122233312221213) & P_{6}:(211333311222222) \\
P_{6}:(212233221312213) & P_{7}:(111222211211212) \\
P_{8}:(221444322322334) & P_{8}:(222344412432323) \\
P_{8}:(222344432412323) & P_{8}:(223444322322314) \\
P_{10}:(111222011211112) & P_{10}:(111222211011112) \\
P_{10}:(111022211211112) & P_{10}:(111222211211110)
\end{array}
$$

The other four types with 24 extreme rays are three-dimensional, and each of them has three-dimensional prime metrics among its extreme rays. For instance, the following 18 vectors are extreme rays of the cone \# 338:

$$
\begin{array}{cc}
3 / 3 \text { split }:(110010110110011) & 3 / 3 \text { split }:(101011010101101) \\
3 / 3 \text { split }:(010111011100110) & 3 / 3 \text { split }:(001110111111000) \\
P_{1}:(111122221221211) & P_{4}:(111121111211111) \\
P_{5}:(322243331422222) & P_{5}:(222142232432323) \\
P_{5}:(122243333422222) & P_{5}:(222342232432321) \\
P_{9}:(221232121321121) & P_{9}:(121131222311222) \\
P_{9}:(112132122322112) & P_{9}:(212231221312211) \\
P_{11}:(111122221221011) & P_{11}:(111122221201211) \\
P_{11}:(111120221221211) & P_{11}:(111122021221211)
\end{array}
$$

The corresponding lists for all 339 types appear on our website.

It is instructive to draw the tight spans of the eleven prime metrics. Four of them are actually three-dimensional. For instance, the metric $P_{4}$ has the structure of an octahedron. Please compare Figure 5 with [12, Figure 1]. 
For each of the 11 prime metrics, we list the $f$-vector of their tight span:

$\begin{array}{ccccc} & \text { \# vertices } & \text { \# edges } & \text { \# polygons } & \text { \# 3-cells } \\ P_{1}: & 6 & 9 & 4 & 0 \\ P_{2}: & 7 & 15 & 9 & 0 \\ P_{3}: & 6 & 10 & 6 & 1 \\ P_{4}: & 10 & 20 & 12 & 1 \\ P_{5}: & 11 & 20 & 11 & 1 \\ P_{6}: & 7 & 11 & 5 & 0 \\ P_{7}: & 11 & 20 & 10 & 0 \\ P_{8}: & 11 & 19 & 9 & 0 \\ P_{9}: & 7 & 12 & 7 & 1 \\ P_{10}: & 5 & 7 & 3 & 0 \\ P_{11}: & 5 & 7 & 3 & 0\end{array}$

The metrics $P_{1}, \ldots, P_{9}$ each have six trivial vertices in their tight span. The list of non-trivial vertices given in [12, Table 1] is consistent with our list above. The prime metrics $P_{10}$ and $P_{11}$ are improper in the sense that two points have distance zero.

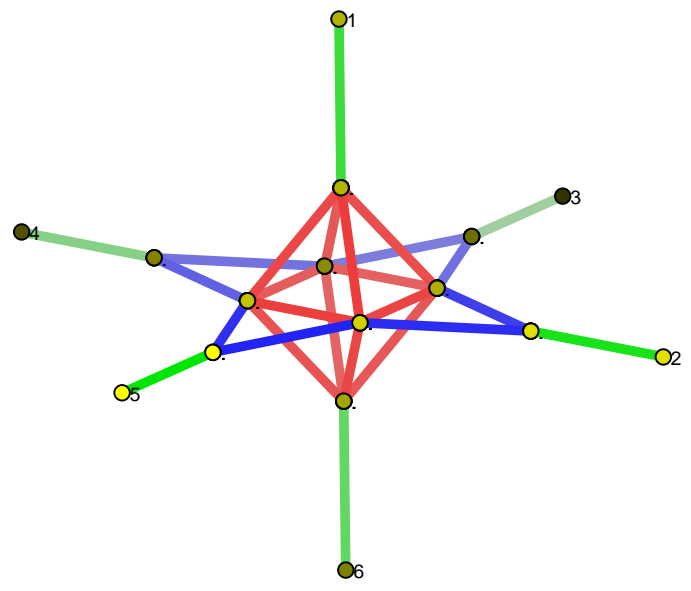

Figure 5: The tight span of the metric $P_{4}+(1,1, \ldots, 1)$

Remark 7 The tight spans of the prime metrics $P_{3}, P_{4}, P_{5}, P_{9}$ have a 3-dimensional cell, while the tight spans of the other seven are 2-dimensional.

The metric fan $M F_{6}$ defines an incidence relation between the prime metrics and the generic metrics. This leads to a finer invariant for distinguishing among the 339 types of generic types. This invariant is the vector $(S, P)=\left(s_{2}, s_{3}, p_{1}, p_{2}, \ldots, p_{11}\right)$ where $p_{i}$ is the number of extreme rays of type $P_{i}$ which lie on the corresponding cone. This invariant resolves about half of the clusters which had been left unresolved by the earlier invariants. 
Example 8 The three two-dimensional types \# 9, \# 10 and \# 11, all have the same invariants $R=(2,8,5), g=2$ and $c=4$ in the list of Section 2. They are distinguished by the new invariant $(S, P)=\left(s_{2}, s_{3}, p_{1}, p_{2}, \ldots, p_{11}\right)$

Type 9 has $(S, P)=(4,0,0,1,0,0,0,2,1,2,0,2,0)$.

Type 10 has $(S, P)=(5,0,0,1,0,0,0,1,2,2,0,2,0)$.

Type 11 has $(S, P)=(5,0,0,1,0,0,0,2,2,2,0,2,0)$.

Example 9 The last three three-dimensional types \# 337, \# 338 and \# 339 all have the same invariants $(f, R, B, S, C)$ in the big table of Section 3. They are distinguished by the new invariant $(S, P)=\left(s_{2}, s_{3}, p_{1}, p_{2}, \ldots, p_{11}\right)$

Type 337 has $(S, P)=(0,4,0,0,1,1,4,0,0,0,4,0,4)$.

Type 338 has $(S, P)=(0,4,1,0,0,1,4,0,0,0,4,0,4)$.

Type 339 has $(S, P)=(0,4,1,0,1,0,4,0,0,0,4,0,4)$.

We close this section with a remark aimed at experts in polytope theory. The metric fan is the secondary fan of the hypersimplex $\Delta(6,2)$, hence it is the normal fan of the secondary polytope $\Sigma(\Delta(6,2))$. Following [2], the vertices of the secondary polytope correspond to the regular triangulations of $\Delta(6,2)$, and the facets of the secondary polytope correspond to minimal regular subdivisions of $\Delta(6,2)$. For instance, Figure 5 is the dual picture to a regular subdivision of $\Delta(6,2)$ into 10 five-dimensional polytopes.

The results described in this section provide the vertex-facet incidence matrix of the secondary polytope $\Sigma(\Delta(6,2))$. In particular, the classification result of Koolen, Moulton and Tönges [12] can be restated as follows.

Corollary 10 Up to the action of the symmetric group on $\{1,2,3,4,5,6\}$, the secondary polytope $\Sigma(\Delta(6,2))$ has precisely 14 facets.

\section{Visualization of Tight Spans in POLYMAKE}

POLYMAKE is a software package developed by Ewgenij Gawrilow and Michael Joswig for studying polytopes and polyhedra [10]. It allows us to define a polyhedron by a set of either inequalities or vertices and computes numerous properties of the polyhedron. We implemented a client program to POLYMAKE for visualizing the tight span $T_{d}$ of a given metric $d$. In short, our program does the following to produce the figures in this paper:

1. Compute all faces of the polyhedron $P_{d}$ from the given metric $d$.

2. Build the tight span $T_{d}$ by extracting the bounded faces of $P_{d}$.

3. Spring-embed the 2-skeleton of $T_{d}$ in 3-space using POLYMAKE's spring embedder and display it using JAVAVIEW.

4. Label the points corresponding to the finite set (the "taxa") on which the metric is defined. In our case, the set of labels is $\{1,2,3,4,5,6\}$. 
The program was developed by the second author with the assistance of Michael Joswig and Julian Pfeifle. The code can be downloaded at

www. math. berkeley. edu/ jyu/.

The figures above only show the combinatorial properties of the tight span and, because of the projection and the spring embedder, the edge lengths seen here do not represent the actual edge lengths in the tight span.

The pictures produced by our software are different from the output produced by the software SPLITSTREE [8]. SPLITSTREE is a program that can, among other things, compute and visualize the split-decompositions of metrics (see $[1,8]$ ). It decomposes an input metric into a sum of splits plus a split-prime metric that cannot be further decomposed into splits. SPLITSTREE outputs a planar graph representing the splitdecomposable part of the input metric, where sets of parallel edges represent splits.

When a metric is split-decomposable, then the tight span is a cubical complex, and the output from SPLITSTREE agrees with our visualization with a few edges removed to make it planar. The edges output by SPLITSTREE are scaled to be metrically accurate. By contrast, our implementation does not preserve the edge lengths. Among the 339 generic metrics on six points, only one type (namely, Type \# 66) is split-decomposable. For all other 338 types, the picture produced by our program contains more refined combinatorial information than the output of SPLITSTREE.

The split-decomposition theory of Bandelt and Dress [1] has the following interpretation in terms of the metric fan. The 31 splits are among the extreme rays of the metric fan. Consider the induced subfan of $M F_{6}$ on the 31 splits. A key result in [1] states that this subcomplex is simplicial, i.e., all cones in this subfan are spanned by linearly independent vectors. Now consider any metric $d$, for instance, one of our 339 generic metrics, and let $C$ be the cone of the metric fan $M F_{6}$ containing $d$ in its relative interior. The intersection of $C$ with the induced subfan of split-decomposable metrics is a simplicial face $F$ of $C$. It follows that $d$ can be written uniquely as a sum of a vector $d^{\prime}$ in $F$ and a positive combination of extreme rays of $C$ not in $F$. The output of SPLITSTREE is the edge graph of the tight span of $d^{\prime}$.

\section{Acknowledgments}

This project grew out of the second author's term project for the Seminar on Mathematics of Phylogenetic Trees which was organized by Lior Pachter and the first author during the Fall Semester 2003 at UC Berkeley. We are grateful to Michael Joswig, Julian Pfeifle and Jörg Rambau for helping us with our computations. Josephine Yu was supported in part by an NSF Graduate Research Fellowship. Bernd Sturmfels was supported by a Hewlett Packard Visiting Research Professorship 2003/2004 at MSRI Berkeley and in part by the NSF (DMS-0200729). 


\section{References}

[1] H.-J. Bandelt and A. Dress: A canonical decomposition theory for metrics on a finite set, Advances in Mathematics 92 (1992) 47-105.

[2] L. Billera, P. Filliman and B. Sturmfels: Constructions and complexity of secondary polytopes, Advances in Mathematics 83 (1990) 155-179.

[3] J. De Loera, B. Sturmfels and R. Thomas: Gröbner bases and the triangulations of the second hypersimplex, Combinatorica 15 (1995) 409-423.

[4] A. Deza, K. Fukuda, T. Misutani and C. Vo: On the face lattice of the metric polytope, Lecture Notes in Computer Science 2866, Springer-Verlag, Berlin, (2003) $118-128$.

[5] M. Deza and M. Laurent: Geometry of Cuts and Metrics, Algorithms and Combinatorics, 15, Springer-Verlag, Berlin, 1997.

[6] A. Dress: Trees, tight extensions of metric spaces, and the cohomological dimension of certain groups, Advances in Mathematics 53 (1984) 321-402.

[7] A. Dress, K. Huber and V. Moulton: Totally split-decomposable metrics of combinatorial dimension two, Annals of Combinatorics 8 (2001) 99-112.

[8] A. Dress, D. Huson and V. Moulton: Analyzing and visualizing sequence and distance data using SplitsTree. Discrete Appl. Math. 71 (1996) 95-109.

[9] M. Dutour: Cut and Metric Cones, Classification of facets and extreme rays for $n \leq 8$ posted at http://www.liga.ens.fr/ dutour/CUT_MET/CutMetricCones.html.

[10] E. Gawrilow and M. Joswig: Polymake: a framework for analyzing convex polytopes. em Polytopes - Combinatorics and Computation (Oberwolfach, 1997), 43-73, DMV Sem., 29, Birkhäuser, Basel, 2000.

[11] K. Polthier, S. Khadem, E. Preuss, and U. Reitebuch: JAVAVIEW: Interactive 3D Geometry and Visualization, www. javaview.de.

[12] J. Koolen, V. Moulton and U. Tönges: A classification of the six-point prime metrics, European Journal of Combinatorics 21 (2000) 815-829.

[13] J. Rambau: TOPCOM: triangulations of point configurations and oriented matroids. Mathematical Software (Beijing, 2002), 330-340, World Sci. Publishing, River Edge, NJ, 2002. 